\title{
Combined quantum mechanical and molecular mechanical methods to study chemical events in complex biological environments
} M Elstner

\author{
Address: Institut für Physikalische und Theoretische Chemie, TU Braunschweig, Germany \\ from 4th German Conference on Chemoinformatics \\ Goslar, Germany. 9-II November 2008 \\ Published: 5 June 2009 \\ Chemistry Central Journal 2009, 3(Suppl I):O2I doi:I0.II86/I752-I53X-3-SI-O2I
}

This abstract is available from: http://www.journal.chemistrycentral.com/content/3/SI/O2I

(C) 2009 Elstner; licensee BioMed Central Ltd.

Biological systems make use of fascinating 'molecular machines' to catalyse chemical reactions or interchange different forms of energy. This is done with an efficiency unmatched by artificial systems. E.g., the conversion of light into chemical energy occurs within sub-picosecond time-scales and with quantum yields exceeding $60 \%$.

These complex structures and processes pose a big challenge for the computational approaches, developed in Physics, Chemistry and Biology. The occurring length and time scales can not easily be treated by any existing method. Therefore, in the last years considerable effort has been put forward to combine different methods in so called multi-scale approaches to treat chemical reactions in large systems and in the development of methods, which allow to simulate processes on long time scales. In our work, we mainly focussed on the development of an approximate Density Functional method (DFTB: Density Functional Tight Binding) and its integration into a multiscale framework, including combined quantum mechani$\mathrm{cal} /$ molecular mechanical (QMMM) methods, and a coupling with continuum electrostatic methods (,Multi-scale modelling'). Molecular Dynamics (MD) simulations, reaction path algorithms and methods to calculate free energies allow detailed insights into dynamic and thermodynamic properties of biomolecular systems.

Our main applications focussed onto proton transfer reactions and photochemical processes. Proton transfer is a ubiquitous elementary step in enzymatic reactions, in particular in bioenergetics. In photosynthesis, the energy of photons is used to create a $\mathrm{pH}$ gradient by proton translocation across the membrane, which is transformed into chemical energy (ATP) in succeeding steps. An instructive example is the protein Bacteriorhodopsin. Its chromophor features a very efficient conversion of the energy of the photon and ultrafast switching times, which is caused by the specific interaction of the chromophore with the protein environment. We discuss the processes after photoabsorption, several steps in the proton translocation along the photo-cycle and the mechanism of colour tuning in Rhodopsins in general, which are also the biophysical basis for colour vision in mammals. 